\section{Magnetic resonance and GPs decisions}

I wish to comment on this paper, reporting the results of the DAMASK trial, ${ }^{1}$ in which I and my local colleagues participated as orthopaedic surgeons.

The paper seems to leave many unanswered questions. There is little or no detail on what orthopaedic treatment was undertaken in either group, or whether the diagnoses made were definitive or speculative.

What is clear is that the change on diagnosis by MRI and orthopaedic consultation was about the same, but that GPs had more confidence in the MRI diagnosis than in the orthopaedic diagnosis. This is an important finding and deserves more attention.

Our GP colleagues do not trust us, and would rather trust an MRI. We could argue the appropriateness of that position with regard clinical diagnosis - MRls are not always right, and are every bit as operatordependant as a clinical orthopaedic examination. That lack of faith would be bad enough, but if it is based upon surgical findings and GPs have more faith in an MRI than in the results of an arthroscopy, then orthopaedic surgeons have a real problem. The findings of this study, as discussed, are bland and have completely ignored an extremely important and contentious issue that deserves further comment.

\author{
Alasdair Sutherland \\ Clinical Senior Lecturer/Hon Consultant \\ Director, North of Scotland Orthopaedic \\ Training Programme, Department of \\ Orthopaedics, University of Aberdeen, \\ Aberdeen, AB25 2ZD. \\ E-mail ort025@abdn.ac.uk
}

\section{REFERENCES}

1. Damask Trial Team. Influence of magnetic resonance imaging of the knee on GPs' decisions: a randomised trial. Br J Gen Pract 2007; 57(541): 622-629.

10.3399/bjgp08X263848

\section{Authors' response}

We thank you for your comments. In our randomised trial we assessed the effect of early access to MRI compared with referral to an orthopaedic specialist on GPs' diagnoses and treatment plans for patients with knee problems. Therefore, the paper presents what were the treatment plans that GPs used and not that of the orthopaedic specialist.

Similarly, the diagnoses recorded were that of the GPs and we must therefore assume that these diagnoses were as definitive as what the GPs thought at the time. It was not the objective of the paper to examine the effect of MRI or not on orthopaedic management, but as part of the broader project we collected data on the proportion of arthroscopies performed in each trial arm. There is evidence that a significantly higher proportion of patients in the MRI group had an arthroscopy. Furthermore, we found that the proportion of patients who had a diagnostic arthroscopy was significantly higher in the orthopaedic referral group. GP access to MRI, therefore, did significantly affect orthopaedic specialist treatment plans in terms of the use of arthroscopies, which is presented in more detail in a further paper.

The evidence from our trial does show that GPs were more confident in their diagnoses and treatment plans when MRI was accessible to them. The effect of MRI findings on GPs' decisions, however, was compared with the effect of the orthopaedic specialist letter after initial consultation with the patient. Therefore, GPs were not recording their diagnoses or management plans based on surgical findings from arthroscopy. In the paper, we provide reasons why GPs might be more confident in the report of a radiologist:

- differences in the content of an MRI report compared with an orthopaedic referral letter;

- early access to MRI provides greater continuity in care as a result of the patient receiving an intervention earlier in the care pathway; and

- evidence for other body areas, such as the lumbar spine, where GPs request radiographs to reassure themselves and their patients.

Ultimately, despite the significant increase in confidence, GPs did not diagnose or treat patients differently on the basis of an MRI report compared with an orthopaedic specialist letter. Interestingly, as described above, it appears that the availability of the MRI examinations had more of an effect on orthopaedic specialists' practice.

\section{Stephen Brealey on behalf of the DAMASK trial team}

Department of Health Sciences, Seebohm Rowntree Building, University of York, York Y010 5DD. E-mail: sb143@york.ac.uk

10.3399/bjgp08X263857

\section{Correction}

In the letter 'Supporting self-care in general practice'. Br J Gen Pract 2007; 57(545): 996, two of the authors were omitted, Rachel Conroy and Reena Doshi. Please accept our apologies for this error. The correct version of the letter is available online.

10.3399/bjgp08X263875 